\title{
Optimized cultivation of porcine choroid plexus epithelial cells, a blood-cerebrospinal fluid barrier model, for studying granulocyte transmigration
}

\author{
Alexa N. Lauer ${ }^{1} \cdot$ Martin März $^{1} \cdot$ Svenja Meyer $^{1} \cdot$ Marita Meurer $^{2,3} \cdot$ Nicole de Buhr $^{2,3} \cdot$ Julia Borkowski $^{1} \cdot$

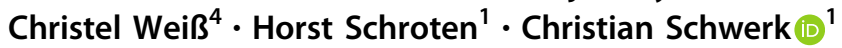

Received: 15 November 2018 / Revised: 13 February 2019 / Accepted: 25 February 2019 / Published online: 17 April 2019

(c) United States \& Canadian Academy of Pathology 2019

\begin{abstract}
The blood-cerebrospinal fluid barrier (BCSFB) plays important roles during the transport of substances into the brain, the pathogenesis of central nervous system (CNS) diseases, and neuro-immunological processes. Along these lines, transmigration of granulocytes across the blood-cerebrospinal fluid (CSF) barrier (BCSFB) is a hallmark of inflammatory events in the CNS. Choroid plexus (CP) epithelial cells are an important tool to generate in vitro models of the BCSFB. A porcine CP epithelial cell line (PCP-R) has been shown to present properties of the BCSFB, including a strong barrier function, when cultivated on cell culture filter inserts containing a membrane with $0.4 \mu \mathrm{m}$ pore size. For optimal analysis of pathogen and host immune cell interactions with the basolateral side of the CP epithelium, which presents the physiologically relevant "blood side", the CP epithelial cells need to be grown on the lower face of the filter in an inverted cell culture insert model, with the supporting membrane possessing a pore size of at least $3.0 \mu \mathrm{m}$. Here, we demonstrate that PCP-R cells cultivated in the inverted model on filter support membranes with a pore size of $3.0 \mu \mathrm{m}$ following a "conventional" protocol grow through the pores and cross the membrane, forming a second layer on the upper face. Therefore, we developed a cell cultivation protocol, which strongly reduces crossing of the membrane by the cells. Under these conditions, PCP-R cells retain important properties of a BCSFB model, as was observed by the formation of continuous tight junctions and a strong barrier function demonstrated by a high transepithelial electrical resistance and a low permeability for macromolecules. Importantly, compared with the conventional cultivation conditions, our optimized model allows improved investigations of porcine granulocyte transmigration across the PCP-R cell layer.
\end{abstract}

\section{Introduction}

Located in the brain ventricles, the choroid plexus (CP), which produces the majority of the cerebrospinal fluid

Christian Schwerk

christian.schwerk@medma.uni-heidelberg.de

1 Department of Pediatrics, Pediatric Infectious Diseases, Medical Faculty Mannheim, Heidelberg University, Mannheim, Germany

2 Department of Physiological Chemistry, University of Veterinary Medicine Hannover, Hannover, Germany

3 Research Center for Emerging Infections and Zoonoses (RIZ), University of Veterinary Medicine Hannover, Hannover, Germany

4 Institute of Medical Statistics and Biomathematics, Medical Faculty Mannheim, Heidelberg University, Mannheim, Germany
(CSF), presents a highly vascularized structure consisting of endothelial and epithelial cells. The most notable feature of the $\mathrm{CP}$ epithelium is the formation of tight junctions, which, in vivo, are required for a major barrier to the central nervous system (CNS). Additional features of the $\mathrm{CP}$ in vivo include fenestrated endothelial cells, which line the capillaries in the $\mathrm{CP}$, and a stroma containing immune cells responsible for immune surveillance. This overall structure and cell arrangement forms the blood-CSF barrier (BCSFB) in vivo $[1,2]$. The BCSFB plays an important role during inflammation of the CNS due to the entry of polymorphonuclear neutrophils (PMNs) into the brain across the $\mathrm{CP}$ epithelium [3-5].

Numerous in vitro $\mathrm{CP}$ epithelial cell lines and primary cells exist [6]. To imitate a cellular barrier in vitro, $\mathrm{CP}$ epithelial cells are usually grown on cell culture filter inserts, which allows the generation of a cell culture 
system consisting of two compartments separated by a cell layer grown on a permeable support. Such in vitro models are important for, e.g., the analysis of pharmaceutical agents that have to be delivered to the CNS, but also for studying microbial agents which utilize the $\mathrm{CP}$ as an entry gate [6-9]. In addition, immune cell interactions with and migration across host cells can be investigated using these in vitro barrier models [10]. Importantly, CP epithelial cells can be cultivated either in standard or inverted filter culture systems. In the standard model, the cells are cultivated on the upper face of the filter support with the basolateral cell side facing towards the lower compartment. In contrast, in the inverted model the cells are grown on the lower face of the membrane. In this case the basolateral side of the cells is directed towards the upper compartment of the filter insert. These two distinct in vitro models enable studies of host cell interactions and responses when exposed to pathogens, immune cells, or chemical agents from the apical (in vivo-CSF), as well as the basolateral (in vivo-blood) side [9, 11, 12].

Immortalized porcine choroid plexus epithelial cells (PCP-R) were first characterized in our laboratory by Schroten and colleagues [13]. PCP-R cells originated from primary porcine choroid plexus epithelial cells (PCPEC) via a series of sub-cultivation steps and display similar morphological and physiological features to the primary cells. In our previous experiments, PCP-R cells were cultivated via the standard method on cell culture filter inserts with a pore size of $0.4 \mu \mathrm{m}$ [13]. When the experimental aim is directed towards the analysis of the interaction with and transmigration of pathogens or immune cells through the CP epithelial cells from the basolateral blood side, cell cultivation in the inverted model using filters with a pore diameter of at least $3.0 \mu \mathrm{m}$ is required. However, cultivation of cell layers on filter inserts with a pore size of $3.0 \mu \mathrm{m}$ or larger can result in cell growth through the filter membrane [14].

Similarly, when PCP-R cells were cultivated on filters with a pore size of $3.0 \mu \mathrm{m}$ according to previously described methods $[11,15]$, undesired growth of the cells through pores of the filter membrane was encountered during this study. Therefore, our aim was to develop a protocol which allows cultivation of PCP-R cells in the inverted model on filters with $3.0 \mu \mathrm{m}$ pore diameter with minimal growth of the cells through the filter membrane. Here, we describe an optimized cell culture method for PCP-R cells, which results in a strong barrier and a strongly reduced growth through the filter. Furthermore, in a practical application we demonstrate an improved transmigration of porcine granulocytes when applying the optimized culture conditions.

\section{Materials and Methods}

\section{Cells and media}

PCP-R cell culture was performed as described previously [13], with the following modifications. The cells were cultivated in DMEM containing $4.5 \mathrm{gl}^{-1}$ glucose, pyruvate, and phenol red (GlutaMAX ${ }^{\mathrm{TM}}$, Gibco by Life Technologies, Carlsbad, USA) and supplemented with $10 \%$ (v/v) fetal calf serum (FCS; Gibco by Life Technologies, Carlsbad, USA), $2 \%$ (v/v) Penicillin-Streptomycin (P/S; $5000 \mathrm{U} \mathrm{ml}^{-1}$ stock; Gibco by Life Technologies, Carlsbad, USA), 2.4\% (v/v) HEPES (1 M stock; Lonza, Basel, Switzerland), $0.05 \%$ (v/v) recombinant human insulin solution $\left(10 \mathrm{mg} \mathrm{ml}^{-1}\right.$ stock; Sigma-Aldrich, St. Louis, MO, USA). This medium composition will be referred to as $10 \%$ FCS PCP-R medium.

\section{Growth of PCP-R cells on cell culture filter inserts}

\section{"Conventional" protocol}

The PCP-R cells were seeded with a density of 400,000 cells per filter onto the inverted cell culture filter inserts (pore diameter $3.0 \mu \mathrm{m}$, pore density $2 \times 10^{6}$ pores per $\mathrm{cm}^{2}$, surface area $0.33 \mathrm{~cm}^{2}$; Greiner Bio-One 662631 Thincert Cell Culture Insert, Kremsmünster, Austria) and incubated for $4 \mathrm{~h}$. The filters were then inverted in a 24-multiwell cell culture plate, which supplied the PCP-R cells with $10 \%$ FCS PCP-R medium. The PCP-R 10\% FCS medium was exchanged daily post-seeding. Once the PCP-R cells reached a TEER of at least $50 \Omega \times \mathrm{cm}^{2}$, the cells were changed to phenol red-free DMEM containing $4.5 \mathrm{gl}^{-1}$ glucose (Gibco by Life Technologies, Carlsbad, USA) and supplemented with $2 \%$ (v/v) P/S, 2.4\% (v/v) HEPES, 2\% glutamine $(200 \mathrm{mM}$ stock; Gibco by Life Technologies, Carlsbad, USA), $1.2 \%$ (v/v) sodium pyruvate $(100 \mathrm{mM}$ stock; Gibco by Life Technologies, Carlsbad, USA), $0.05 \%$ (v/v) recombinant human insulin solution. This medium composition will be referred to as serum-free medium (SFM). All incubation steps were carried out in a humidified incubator at $37^{\circ} \mathrm{C}$ and $5 \% \mathrm{CO}_{2}$.

\section{Collagen coating}

In order to cultivate cells on collagen-coated cell culture filter inserts, $5 \mu \mathrm{g} \mathrm{cm}^{2-1}$ of rat collagen I, lower viscosity, (3 $\mathrm{mg} \mathrm{ml}^{-1}$ stock; R\&D Systems, Minneapolis, MN, USA) was used to coat the filter membrane. The coated filters were incubated for $1 \mathrm{~h}$ in a humidified incubator at $37^{\circ} \mathrm{C}$ and $5 \% \mathrm{CO}_{2}$. Subsequently, the filters were rinsed twice with DPBS (Gibco by Life Technologies, Carlsbad, USA) before the PCP-R cell suspension was applied. Seeding and 
cultivation of the cells was performed as described for the "conventional" protocol.

\section{Application of Parafilm ${ }^{\mathrm{TM}}$}

In order to apply Parafilm ${ }^{\mathrm{TM}}$ to the cell culture filter membranes, Parafilm ${ }^{\mathrm{TM}}$ pieces were cut out to fit inside upper filter compartments (apical filter side), which sufficiently covered the filter surface. With the help of a cotton swab, the Parafilm ${ }^{\mathrm{TM}}$ was applied to the apical surface of the cell culture filter, while the basolateral filter surface was held to a hot plate heated to $55^{\circ} \mathrm{C}$, for approximately $10 \mathrm{~s}$. The filters were sterilized by exposing the apical and basolateral filter side to UV-light for 20 min. Following the Parafilm ${ }^{\mathrm{TM}}$ application and sterilization, the PCP-R cells were seeded and cultivated in $10 \%$ FCS PCP-R medium from the apical side only. Using the cells cultivated according to the "conventional" method, which were cultivated in parallel to the Parafilm ${ }^{\mathrm{TM}}$ filters as a control, the Parafilm ${ }^{\mathrm{TM}}$ was removed once the TEER values of the "conventional" cultivated cells reached at least $50 \Omega \times \mathrm{cm}^{2}$. Following the removal of the Parafilm ${ }^{\mathrm{TM}}$ from the filter, $10 \%$ FCS PCP-R medium was added to the basolateral cell side and incubated for at least $30 \mathrm{~min}$ before the TEER was measured. When the TEER was at least $50 \Omega \times \mathrm{cm}^{2}$, the cells were transferred to SFM overnight.

\section{Improved "dry-top" cultivation}

For the improved "dry-top" cultivation method, seeding and cultivating of the cells was performed as described for the "conventional" protocol, with the following modifications. Following inversion into the 24-multiwell cell culture plate, the PCP-R cells were supplied only from the apical cell side with $600 \mu \mathrm{l}$ of $10 \%$ FCS medium. No medium was added to the basolateral cell side (upper side of the filter compartment) post-seeding. When the cells reached a TEER value of at least $50 \Omega \times \mathrm{cm}^{2}$, the PCP-R cells, which upto this point were supplied with medium only from the apical side, received $10 \%$ FCS-containing PCP-R medium also from the basolateral side and were incubated for $30 \mathrm{~min}$. Following the 30 min FCS exposure, the medium was removed and the cells were placed in SFM overnight. Again, all incubation steps were carried out in a humidified incubator at $37^{\circ} \mathrm{C}$ and $5 \% \mathrm{CO}_{2}$.

\section{Measurement of transepithelial electrical resistance (TEER)}

The TEER of PCP-R cells was measured as previously described [13]. In brief, a voltohmmeter using the STX-2 electrode system (Millipore, Schwalbach, Germany) was used to measure the TEER of the epithelial cells grown on the cell culture filter membranes. Experiments were performed at least three times in at least triplicates.

\section{Determination of paracellular permeability}

To monitor the PCP-R paracellular permeability, Texas Red $^{\mathrm{TM}}$-labeled dextran (3000 MW; Invitrogen ${ }^{\mathrm{TM}}$, Paisley, UK) was added to the upper compartment and measured in the lower compartment after the indicated amount of time as previously described [12]. Based on a standard curve, the percentage of the dextran flux could be calculated. Values are given in mean \pm standard deviation. Experiments were performed three times in at least duplicate.

\section{Immunofluorescent staining and microscopy}

Visualization of nucleus, actin cytoskeleton, and the tight junction protein ZO-1 in PCP-R cells was done as described by Schroten and colleagues [13] with the following modifications. The PCP-R cells were permeabilized with DPBS (without $\mathrm{Ca}^{2+}$ and $\mathrm{Mg}^{2+}$; Gibco by Life Technologies, Carlsbad, USA) containing $0.1 \%(\mathrm{v} / \mathrm{v})$ Triton X-100 (Sigma-Aldrich, Steinheim, Germany) and $1 \%(\mathrm{w} / \mathrm{v})$ bovine serum albumin (BSA; Merck, Darmstadt, Germany) for $10 \mathrm{~min}$ at room temperature. The cell layer was rinsed twice and blocked for $20 \mathrm{~min}$ in DPBS containing 1\% BSA. The secondary antibody Chicken anti-Rabbit Alexa 594 (2 $\mathrm{mg} \mathrm{ml}^{-1}$; Molecular Probes, Oregon, USA), Alexa Fluor $^{\mathrm{TM}} 488$ Phalloidin (300 U; Molecular Probes, Oregon, USA), and 4'-6'-diamidino-2-phenylindole dihydrochloride (DAPI; Invitrogen ${ }^{\mathrm{TM}}$ Molecular Probes ${ }^{\mathrm{TM}}$, Carlsbad, CA, USA) were diluted 1:200, 1:250, and 1:50,000, respectively, in DPBS containing 1\% BSA and incubated for $1 \mathrm{~h}$ at room temperature in the dark. Following the secondary antibody, Alexa Fluor ${ }^{\mathrm{TM}} 488$ Phalloidin, and DAPI incubation, the filters were rinsed three times in DPBS (without $\mathrm{Ca}^{2+}$ and $\mathrm{Mg}^{2+}$ ) before being embedded in ProLong Antifade Reagent (Invitrogen ${ }^{\mathrm{TM}}$ Molecular Probes ${ }^{\mathrm{TM}}$, Carlsbad, CA, USA).

Following the transmigration assay, a representative filter for each condition was fixed and stained for analysis. The staining protocol was followed as described above, with the exception that, instead of Alexa Fluor ${ }^{\mathrm{TM}} 488$ Phalloidin, Alexa Fluor ${ }^{\mathrm{TM}} 660$ Phalloidin (Invitrogen ${ }^{\mathrm{TM}}$ Molecular Probes $^{\mathrm{TM}}$, Carlsbad, CA, USA) was used to stain the actin cytoskeleton, to prevent overlap of the fluorescent emission/ excitation of cell tracker green (CTG) used to label the granulocytes (see below).

To analyze and capture the immunofluorescent images, a Zeiss AxioObserver Z1 equipped with an Apotome $^{\circledR}$ and a $\times 63 / 1.4$ objective lens and the Zeiss Axiovision software (Carl Zeiss, Jena, Germany) were utilized. 


\section{Isolation of porcine granulocytes}

Fresh porcine blood, which was collected in $9 \mathrm{ml}$ lithium heparin tubes (S-Monovette Sarstedt, Nümbrecht, Germany) from healthy pigs, was mixed with 1 volume of LPS-free DPBS (with $\mathrm{Ca}^{2+}$ and $\mathrm{Mg}^{2+}$ ). In a $50 \mathrm{ml}$ conical tube, $12 \mathrm{ml}$ Biocoll Separation Solution $\left(1.077 \mathrm{~g} \mathrm{ml}^{-1}\right.$; Biochrom, Merck, Darmstadt, Germany) was added to the bottom and $12 \mathrm{ml}$ of the blood-PBS mixture was gently added on top of the Biocoll solution and centrifuged at $400 \times g$ for $20 \mathrm{~min}$ and $20^{\circ} \mathrm{C}$ without a rotor brake following the centrifugation. The supernatant above the red bottom layer was discarded and the red layer was suspended in $8 \mathrm{ml}$ of ice cold $0.2 \%$ sodium chloride in the conical tube for erythrocyte lysis, inverted for $30 \mathrm{~s}$, and subsequently $8 \mathrm{ml}$ of ice cold $1.6 \%$ sodium chloride was added. The cell suspension was centrifuged at $250 \times g$, for $7 \mathrm{~min}$ at $4{ }^{\circ} \mathrm{C}$ using a rotor brake following centrifugation. The supernatant was discarded, and the erythrocyte lysis step was repeated a maximum of 2 times, until the remaining cell pellet was white. The white pellet was re-suspended in $10 \mathrm{ml}$ fresh SFM (DMEM without phenol red) and a final concentration of $1.5 \mu \mathrm{M}$ of CellTracker ${ }^{\mathrm{TM}}$ Green CMFDA Dye (CTG; Thermo Fisher Scientific, Waltham, MA, USA) was added to the granulocytes according to the manufacturer's instructions. Lastly, the granulocyte cell suspension was centrifuged at $50 \times g$ for $10 \mathrm{~min}$, the supernatant was removed, and the cell density was adjusted in PCP-R $1 \%$ FCS medium (see below).

\section{Porcine granulocyte transmigration assay}

For the transmigration assay, SFM medium containing $1 \%$ FCS was used and will be referred to as $1 \%$ FCS medium. The assay was carried out in a 24-multiwell cell culture plate (Greiner Bio-One, Kremsmünster, Austria). In the bottom of each well (apical side of the PCP-R cells), $1 \mathrm{ml}$ of PCP-R $1 \%$ FCS medium with either no cytokines, recombinant porcine interleukin-8 (IL-8; R\&D Systems, Minneapolis, Minnesota, USA) with a final concentration of $100 \mathrm{ng} \mathrm{ml}^{-1}$, or recombinant porcine Tumor Necrosis Factor alpha (TNF $\alpha$; R\&D Systems, Minneapolis, Minnesota, USA) with a final concentration of $100 \mathrm{ng} \mathrm{ml}^{-1}$ was added. To the upper compartment (basolateral PCP-R cell side) of the filter insert, 400,000 CTG-labeled porcine granulocytes, which were suspended in PCP-R 1\% FCS medium, and a final concentration of $0.05 \mathrm{mg}$ of Dextran Texas Red ${ }^{\mathrm{TM}}$ were added per filter. The granulocytes were left to transmigrate across the confluent PCP-R cell layer for $4 \mathrm{~h}$ at $37{ }^{\circ} \mathrm{C}$ and $5 \% \mathrm{CO}_{2}$. Following the incubation period, the transmigrated granulocytes were counted via Flow Cytometry.

Flow Cytometry analysis was performed by using Attune ${ }^{\circledR}$ NxT Acoustic Focusing Flow Cytometer (Laser $488 \mathrm{nM}$ $(50 \mathrm{~mW})$, filter $\mathrm{BL} 1=530 / 3$; Thermo Fisher Scientific,
Waltham, MA, USA) with following setup: The threshold was adjusted to a 1:2 mixture of stained and unstained cells. A total draw volume of $50 \mu \mathrm{l}$ was used and the acquisition speed was set to $200 \mu \mathrm{l} \mathrm{min}{ }^{-1}$, with all events being recorded. For determination of granulocytes, the gate was set based on FSC and SSC to exclude cell debris.

Cell tracker fluorescence was collected in the BL1 channel. The measured cell density in $50 \mu \mathrm{l}$ was multiplied by 20 in order to obtain the total number of transmigrated granulocytes in $1 \mathrm{ml}$. All samples were measured twice and a mean was calculated. A standard curve using $4 \times 10^{4}, 2 \times 10^{5}$, and $4 \times 10^{5}$ granulocytes $\mathrm{ml}^{-1}$ was used in order to calculate the percentage of transmigrated granulocytes for each experiment.

The transmigration assay was carried out at least three times in triplicate. One filter of each condition was fixed, stained, and analyzed with the fluorescence microscope to ensure that no extensive cellular growth through the filter membrane occurred during the $4 \mathrm{~h}$ transmigration assay, which was carried out in $1 \%$ FCS media (data not shown).

\section{Animal ethics statement}

Blood was collected from healthy pigs (male and female, German Landrace, various ages) at University of Veterinary Medicine Hannover, which was registered at the Lower Saxonian State Office for Consumer Protection and Food Safety (Niedersächsisches Landesamt für Verbraucherschutz und Lebensmittelsicherheit, No. 18A302), and was conducted in line with the recommendations of the German Society for Laboratory Animal Science (Gesellschaft für Versuchstierkunde) and the German Veterinary Association for the Protection of Animals (Tierärztliche Vereinigung für Tierschutz e. V.) (http://www.gv-solas.de).

\section{Statistics}

The statistical analysis of the transmigrated granulocytes was performed using the SAS system, release 9.4 (SAS Institute Inc., Cary, NC, USA). A one-way ANOVA was used to compare the mean percentages of transmigrated granulocytes in the conventional versus the "dry-top" PCP$\mathrm{R}$ cultivation method. The TEER values are given in mean \pm standard deviation.

\section{Results}

\section{PCP-R cells cross cell culture insert filter membranes with $\mathbf{3 . 0} \mathbf{\mu m}$ pores when cultivated following a "conventional" protocol}

We previously cultivated human and primary PCPEC on inverted cell culture filter models possessing filter membrane 
supports with a pore size of $3.0 \mu \mathrm{m}$ to analyze interaction of pathogens with the cell basolateral side, as well as to investigate transmigration of host immune cells [9, 10]. Although PCP-R cells were previously successfully cultured on filters with $0.4 \mu \mathrm{m}$ pore size [13], growth on membranes with pores of $3.0 \mu \mathrm{m}$ in diameter or larger had not been established yet. Fig. 1a schematically illustrates PCP-R cultivation on inverted filters following previous protocols $[11,15]$. This protocol was adopted with some modifications to filters with $3.0 \mu \mathrm{m}$ pore size as described in Materials and Methods and will be referred to as the "conventional" protocol.

When the "conventional" cultivation method was applied, the cells formed a monolayer with continuous tight junctions,

A

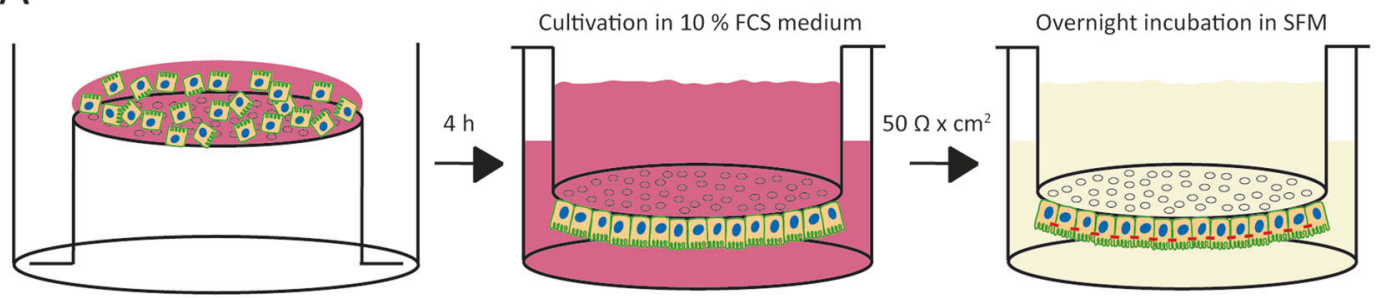

B
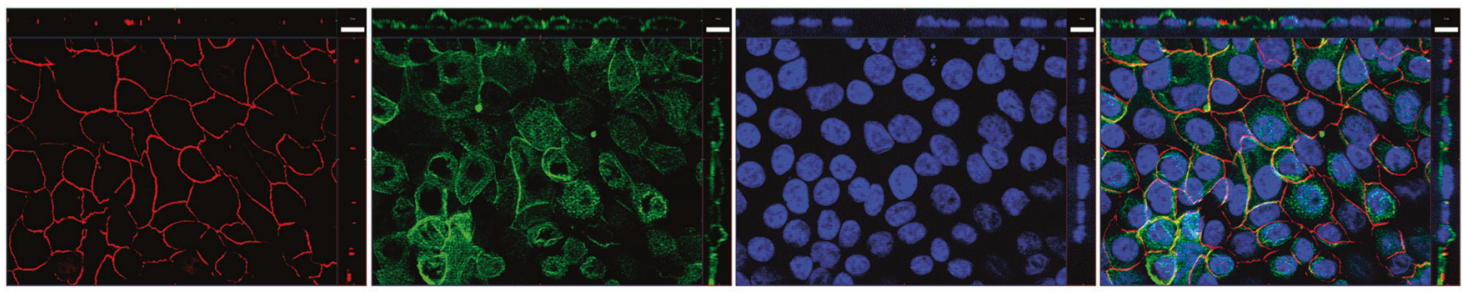

\section{C}
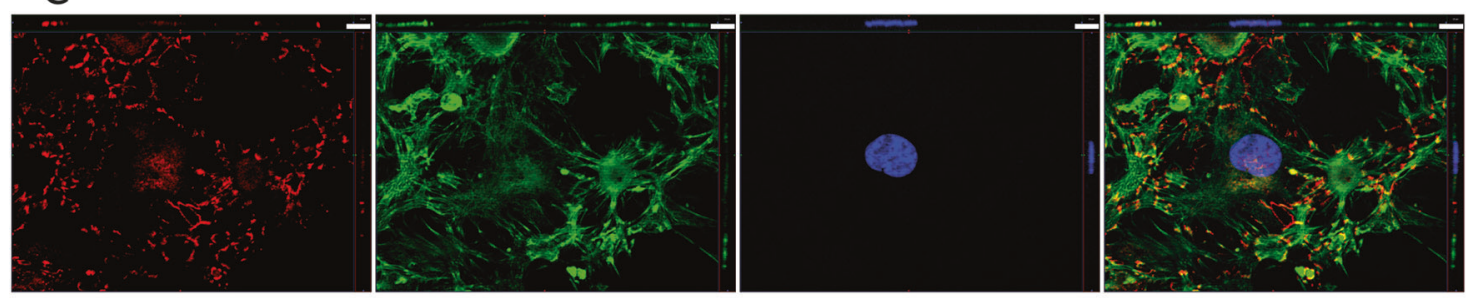

D

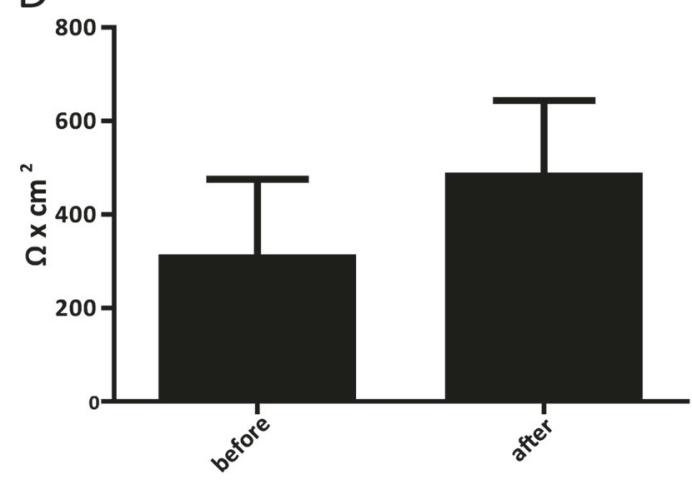

Fig. 1 PCP-R cells cultured following a "conventional" protocol grew through pores with a size of $3.0 \mu \mathrm{m}$ and crossed the filter membranes. a The schematic overview of the "conventional" protocol for culturing PCP-R cells on cell culture filter inserts in an inverted model. b, c ApoTome images of immunofluorescent-stained PCP-R cells. Tight junctions are detected with antibodies against ZO-1 (red); phalloidin was used for staining of the actin cytoskeleton (green), 4,6-diamidino-2-phenylindole for staining of nuclei (blue). Cells found

on the (b) lower face and the (c) upper face of the filter membrane are depicted. The center part of each panel presents a $x y$ en face view of a maximum-intensity projection through the $z$-axis of selected slices. At the top and the right side of each center part are cross-sections through the z-plane of multiple optical slices (basolateral side oriented towards the center part). Size bars indicate $10 \mu \mathrm{M}$. d TEER values developed by the PCP-R cells before and after the switch to serum-free medium (SFM) 
as shown by ZO1 staining, on the filter membrane side, on which they were seeded (Fig. 1b). Noteworthy, under these conditions extensive growth of the cells through the filter pores to the opposite side of the membrane was observed. The observed growth included cellular extensions, which was indicated by staining of the actin filament, and complete cells, as indicated by the detection of DAPI stained nuclei (Fig. 1c). The nuclei density on the opposite filter membrane side was only approximately $10 \%$ compared to the side onto which the cells had been seeded on (data not shown). As expected, the PCP-R cells developed a high TEER when grown following the "conventional" protocol (Fig. 1d). Noteworthy, under these conditions TEER development was very fast and cells were transferred to SFM when presenting TEER values around $250 \Omega \times \mathrm{cm}^{2}$. The percentage of Texas Red ${ }^{\mathrm{TM}}$-labeled dextran flux was determined over a period of $4 \mathrm{~h}$ and was very low $(0.11 \% \pm 0.06)$.

\section{Applying collagen coating and utilizing Parafilm ${ }^{\mathrm{Tm}}$ on the filter membrane to reduce extensive cellular growth through the filter}

In order to limit the growth and crossing of the cells through the filter membrane we attempted different modifications of the cell cultivation protocol. In a first approach we applied a collagen-coating to the filter membrane, as was also attempted by Vandenhaute and colleagues, since it is well known that collagen facilitates cell adherence [14]. Our initial results demonstrated that the PCP-R cells developed a confluent monolayer on the basolateral filter side, however, extensive cellular growth through the filter membrane was still observed. Furthermore, no TEER developed in this cultivation method, which is essential for a proper barrier function (data not shown).

Javaherian and coworkers previously established a method by applying Parafilm ${ }^{\mathrm{TM}}$ to the filter insert membrane in order to achieve micropatterns during polarized epithelial cell growth and maturation in vitro [16-18]. By applying this technique to our model, Parafilm ${ }^{\mathrm{TM}}$ pieces were placed on the inside of the upper filter compartment, as described in the Material and Methods section. This variation of the protocol is schematically depicted in Fig. 2a.

As shown in Fig. 2b, the cells formed monolayers with continuous tight junction strands. Interestingly, we were successful with cultivating the cells on the $3 \mu \mathrm{m}$ pore filter membrane with very limited cell growth through the filter (Fig. 2c), however, no sufficient TEER developed above a basal level with this cultivation method (Fig. 2d). The removal of Parafilm ${ }^{\mathrm{TM}}$ appeared to cause damage to the cell layer on some occasions. Accordingly, the percentage of Texas Red ${ }^{\mathrm{TM}}$-labeled dextran flux, determined over a period of $4 \mathrm{~h}$, was very high with a strong variation $(18.10 \% \pm 9.95)$.

\section{An improved "dry-top" cultivation of PCP-R cells on cell culture filter inserts that facilitates reduced growth of cells through the filter membrane}

"Dry-bottom" protocols have previously been successfully employed to prevent endothelial cells from growing through 3 or $5 \mu \mathrm{m}$ pore size filters. In these protocols the endothelial cells were seeded into the upper compartment and only fed by addition of medium into this compartment, whereas the bottom compartment was left dry [14, 19]. Therefore, we decided to use a "dry-top" protocol, where the PCP-R cells seeded onto the lower face of the filter membrane were only fed by addition of medium into the lower compartment.

When applying this improved "dry-top" cultivation protocol, a cell monolayer with continuous tight junctions, as detected by ZO-1 staining, was formed on the seeding side of the filter membrane (Fig. 3b). Importantly, a strong reduction of cellular growth through the filter membrane pores was observed (Fig. 3c). We found a very limited number of complete cells on the opposite side of the filter membrane, which were indicated by the DAPI-stained nuclei (data not shown).

TEER values were monitored to evaluate the barrier integrity of PCP-R cells cultivated by the "dry-top" protocol. As depicted in Fig. 3d, a high TEER was measured, which was comparable to that observed with the "conventional" method (Fig. 1d). Importantly, the short exposure to medium containing 10\% FCS from the basolateral side was crucial in order for a high TEER to develop with the "drytop" cultivation protocol (data not shown). The percentage of Texas Red ${ }^{\text {TM}}$-labeled dextran flux was determined over a period of $4 \mathrm{~h}$ and was very low $(0.27 \% \pm 0.35)$.

\section{Cultivation of PCP-R cells under optimized conditions results in an increased transmigration of granulocytes}

In the course of CNS inflammation, the BCSFB plays an important role as entry gate for immune cells, including PMNs, into the brain across the CP epithelium [3-5]. In vitro models of the BCSFB, therefore, present important tools to investigate immune cell transmigration towards the CNS.

To demonstrate the advantage of the improved "dry-top" cultivation method in a practical application, transmigration of granulocytes across PCP-R cell layers generated by "drytop" cultivation was compared with that of granulocytes through layers obtained with the "conventional" protocol. Fresh blood-derived porcine granulocytes were applied to the basolateral cell side (upper compartment) in this assay, which mimics the blood side, and the transmigration rate to the apical cell side (bottom compartment) was determined after $4 \mathrm{~h}$. To investigate the effect of cytokines on 

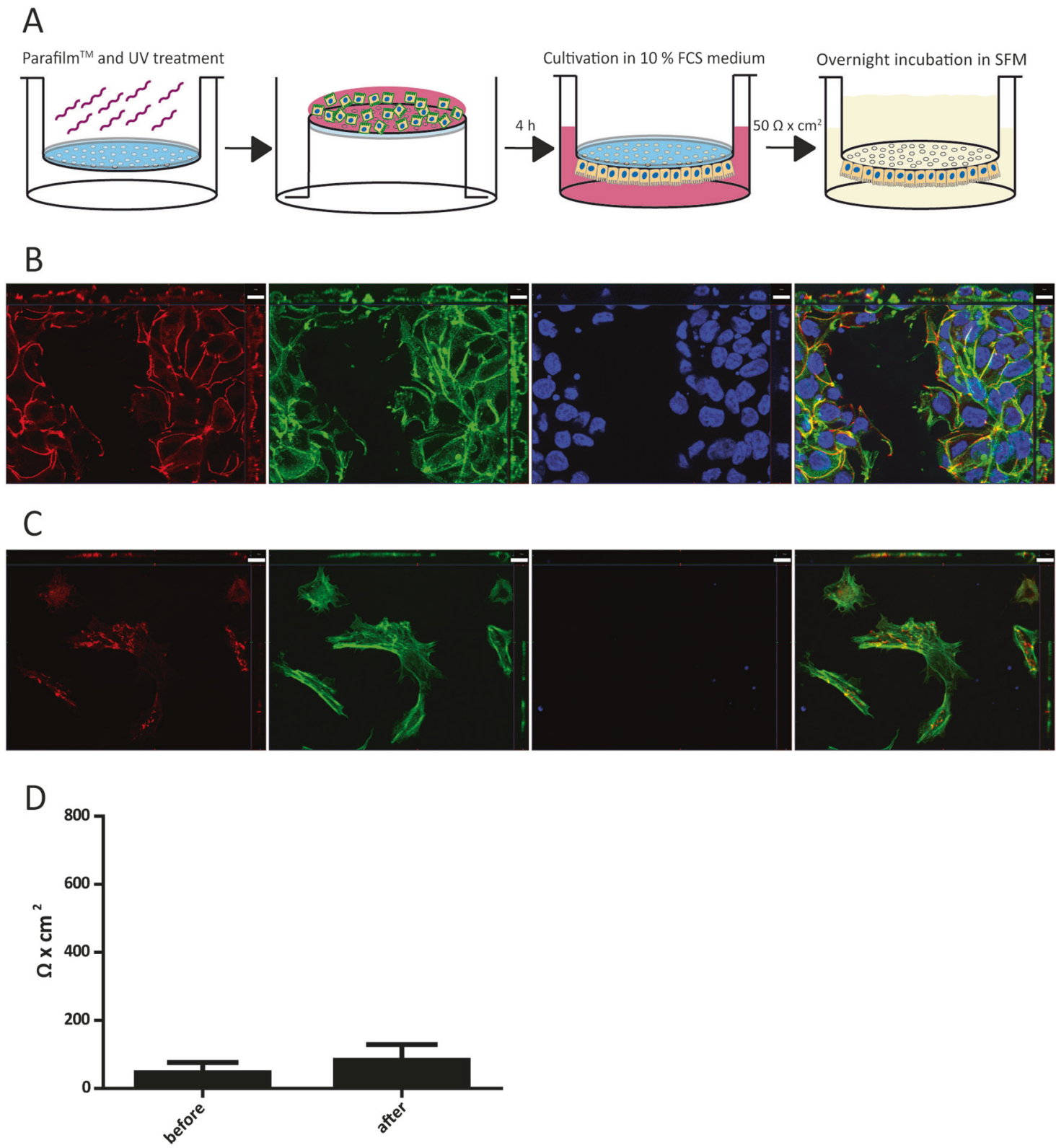

Fig. 2 PCP-R cells cultured following a modified protocol applying Parafilm ${ }^{\mathrm{TM}}$ do not grow through pores with a size of $3.0 \mu \mathrm{m}$ but lack development of a TEER. a Schematic overview of a modified protocol for culturing PCP-R cells on cell culture filter inserts in an inverted model applying Parafilm ${ }^{\mathrm{TM}}$. b, c ApoTome images of immunofluorescence staining of the PCP-R cells. Tight junctions are detected with antibodies against ZO-1 (red); phalloidin was used for staining of the actin cytoskeleton (green), 4,6-diamidino-2-phenylindole for staining of nuclei (blue). Cells found on the (b) lower face and the

granulocyte transmigration, IL- 8 or TNF $\alpha$ were added to the bottom compartment.

During this assay, initial TEER values of cell layers grown by the "conventional" protocol or the "dry-top" cultivation were comparable, with even higher TEER values observed with the "dry-top" filter cultures" (Fig. 4a). During the $4 \mathrm{~h}$ transmigration period high TEER values were

(c) upper face of the filter membrane are depicted. The center part of each panel presents a $x y$ en face view of a maximum-intensity projection through the $z$-axis of selected slices. At the top and the right side of each center part are cross-sections through the z-plane of multiple optical slices (basolateral side oriented towards the center part). Size bars indicate $10 \mu \mathrm{M}$. The lack of cells in the center of (b) seems to be caused by the damage of the cell layer during removal of Parafilm ${ }^{\mathrm{TM}}$. d TEER values developed by the PCP-R cells before and after switch to serum-free medium (SFM)

maintained. Still, TEER values of "dry-top" filter cultures displayed some drop, although final values were comparable to those observed with the "conventional" filter cultures (Fig. 4a).

The percentage of Texas Red ${ }^{\mathrm{TM}}$-labeled dextran flux was determined over a $4 \mathrm{~h}$ period and remained low. The dextran flux values for the "conventional" cultivation were 


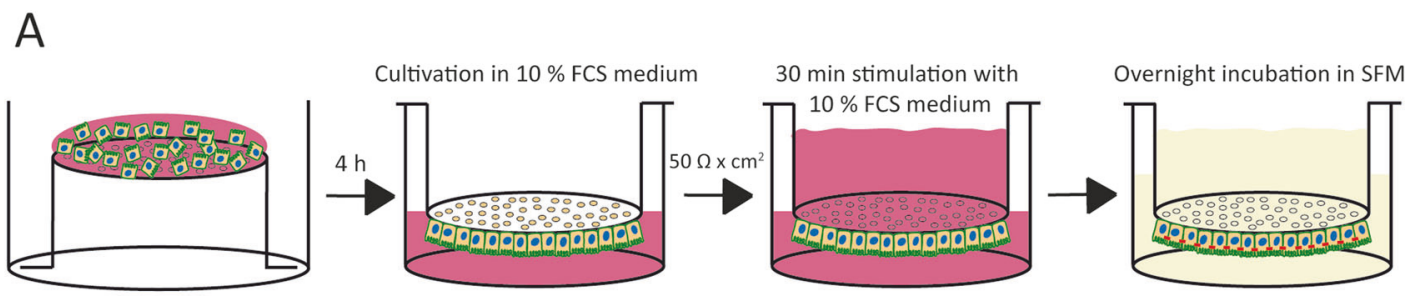

\section{B}
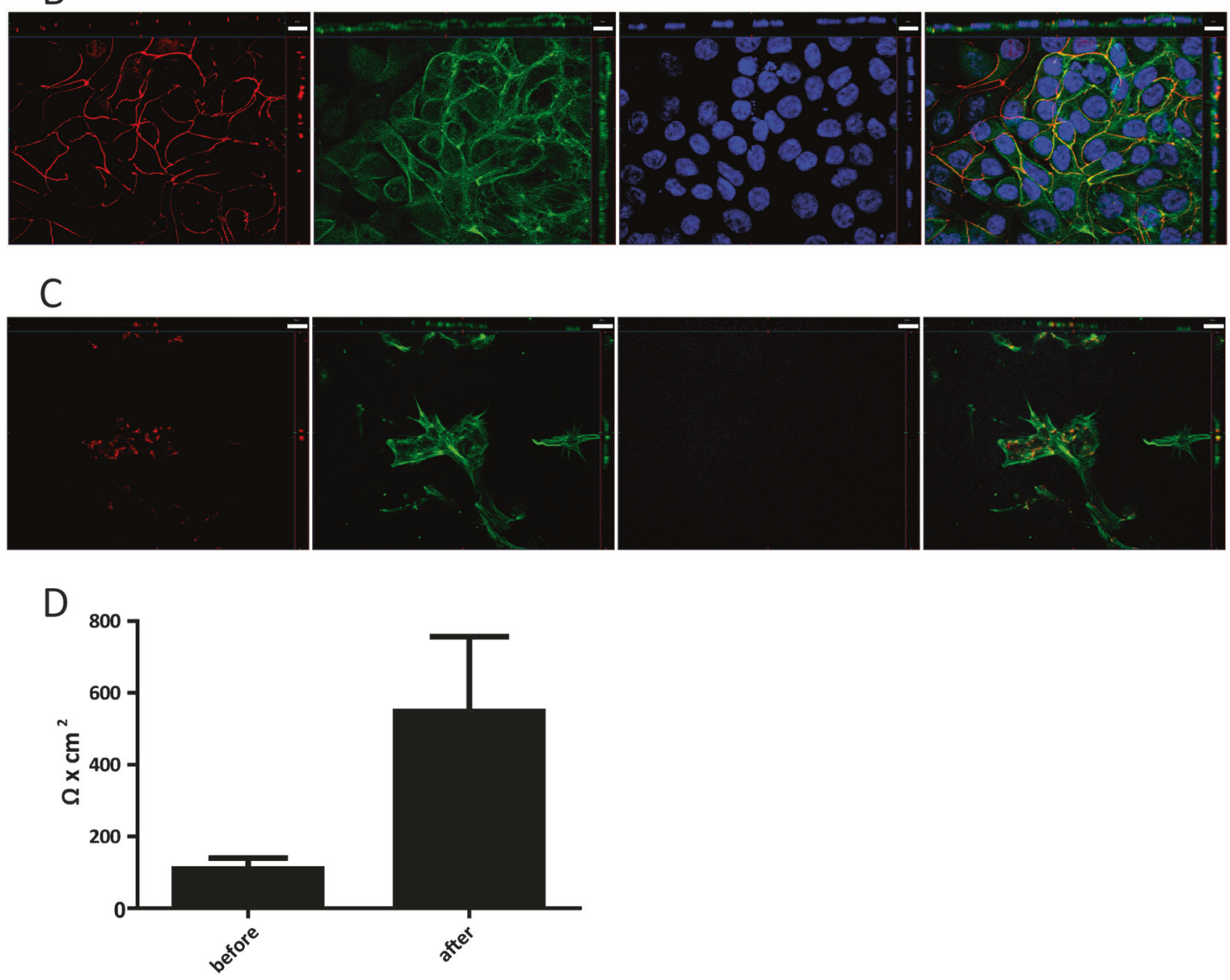

Fig. 3 PCP-R cells cultivated following the improved "dry-top" protocol did not grow through $3.0 \mu \mathrm{m}$ large pores and developed a strong TEER. a The schematic overview of the improved "dry-top" protocol for culturing PCP-R cells on cell culture filter inserts in an inverted model. b, c ApoTome images of immunofluorescent-stained PCP-R cells. Tight junctions are detected with antibodies against ZO-1 (red); phalloidin was used for staining of the actin cytoskeleton (green), 4,6diamidino-2-phenylindole for staining of nuclei (blue). Cells found on

$0.24 \% \pm 0.06$ for the control (no cytokines), $0.29 \% \pm 0.10$ in presence of IL- 8 , and $0.24 \% \pm 0.08$ in presence of TNF $\alpha$, respectively. Values for the "dry-top" cultivation were $0.81 \% \pm 0.55$ for the control (no cytokines), $1.40 \% \pm 1.00$ in presence of IL- 8 , and $1.26 \% \pm 1.00$ in presence of TNF $\alpha$, respectively.

As can be seen in Fig. 4b, granulocytes, which were applied to the "conventionally" cultivated PCP-R cells, exhibited a reduced transmigration rate, regardless of the

the (b) lower face and the (c) upper face of the filter membrane are depicted. The center part of each panel presents the $x y$ en face view of a maximum-intensity projection through the $z$-axis of selected slices. At the top and the right side of each center part are cross-sections through the z-plane of multiple optical slices (basolateral side oriented towards the center part). Size bars indicate $10 \mu \mathrm{M}$. d TEER values developed by the PCP-R cells before and after the switch to serum-free medium (SFM)

presence or absence of IL- 8 or TNF $\alpha$. The average percentage of transmigrated granulocytes which were either not attracted with cytokines, or with the application of the chemoattractants IL- 8 or TNF $\alpha$, was approximately $1 \%$, $36 \%$, and $11 \%$, respectively. In contrast, PCP-R cells cultivated via the improved "dry-top" protocol, which resulted in a drastic decrease in the cell's cytoskeleton growth through the filter membrane, showed an overall higher transmigration rate of the porcine granulocytes (Fig. 4b). 
A

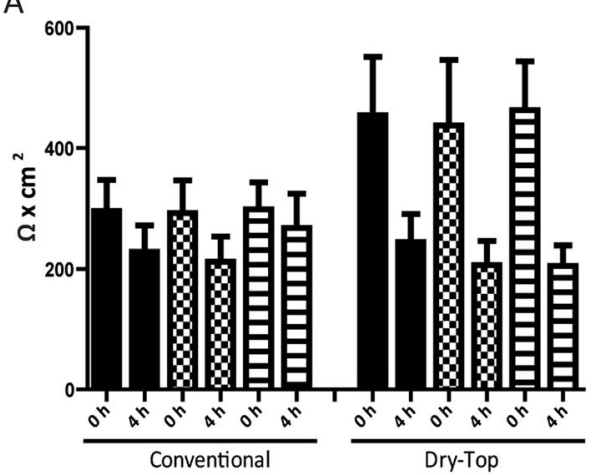

Fig. 4 Cultivating the PCP-R cells via the "dry-top" method results in an overall higher granulocyte transmigration across choroid plexus cell layers. a TEER measurement of the PCP-R layer pre-granulocyte and

Granulocytes, which were not exposed to chemoattractants, exhibited an average transmigration of approximately $8 \%$, which was significant $(p<0.0001)$ compared to the conventional cultivation method. When the chemoattractant IL8 was applied, the transmigration rate was significantly higher $(p<0.0001)$ with an average of $70 \%$ migration. Exposure to TNF $\alpha$ resulted in a significantly higher $(p<$ 0.0018 ) granulocyte transmigration of approximately $28 \%$, which was higher than granulocytes not exposed to a chemotactic stimulant, but still less when compared to cells with IL-8 chemokine treatment.

\section{Discussion}

The BCSFB is constituted by the epithelium of the CP. Hallmarks of in vitro CP epithelial cells are the formation of tight junctions and the presentation of a barrier function characterized by a high TEER. Of special interest for the investigation of the BCSFB are reliable in vitro models, which recapitulate the in vivo situation as faithfully as possible $[1,2,6]$. These models are of high importance for research of inflammatory brain diseases such as meningitis, since the CP has been described as an entry gate of pathogens, as well as host immune cells into the CNS [3-5, 20].

In vitro BCSFB models consisting of immortal or primary CP epithelial cells have been established previously. These include rodent, bovine and porcine model systems [2]. Importantly, the pig has been established as an excellent in vivo model to study the pathogenesis of meningitis. As an example, in contrast to murine and rabbit models, pigs can be intranasally infected with Streptococcus suis ( $S$. suis), reflecting the natural infection route [21]. Porcine in vivo models are used much more often in translational medicine than bovine models [22]. Furthermore, since porcine and human anatomy, physiology and genetics are closely related, the pig is described as one important large animal used for

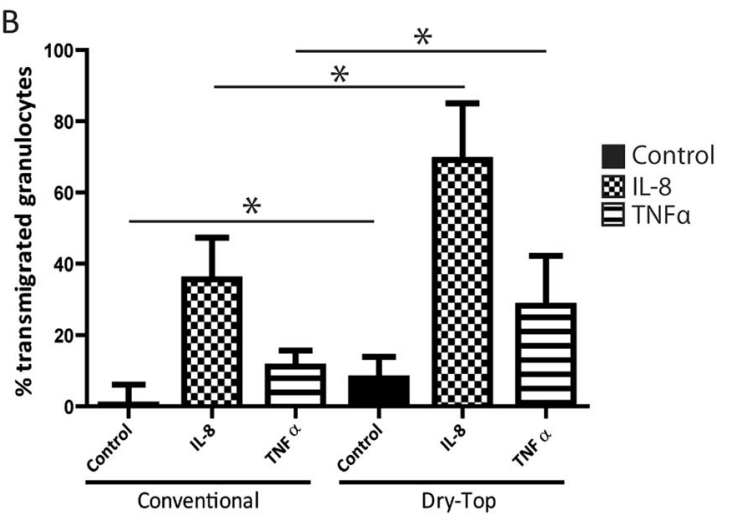

post-granulocyte transmigration. b The average percentage of transmigrated porcine granulocyte through the PCP-R cell layer. Experiments were performed three in times in triplicates; $* p<0.005$

research on human infectious diseases and in translational research instead of dogs and monkeys [23-25]. Noteworthy, techniques for genetic modification have also been described by using minipigs, which allows for the development of disease models based on transgenic animals [26].

Of the available porcine BCSFB models, there are the PCP-R cells, which originated from subcultivated primary porcine $\mathrm{CP}$ epithelial cells and were characterized previously [13]. PCP-R cells have several advantages, since they were not derived from tumor cells, but offer the advantages of immortalized cell lines including much easier availability and handling compared to primary cells. When generated data from porcine in vivo models need to be translated into in vitro models following the $3 \mathrm{R}$ (Replacement, Reduction, Refinement) concept, PCP-R cells, as a porcine in vitro BCSFB model, provide a necessary step for comparison with human in vitro models (e.g. human choroid plexus papilloma epithelial (HIBCPP) cells) [15]. Comparisons between porcine and human models in the context of brain inflammation are important, since human and porcine immune cells can react differently to chemical stimuli, such as phorbol 12-myristate 13-acetate (PMA) [27].

The PCP-R cells, however, had not yet been established in a functional inverted cell culture model with the use of filter membranes with a pore diameter of at least $3.0 \mu \mathrm{m}$. Attempts to cultivate these cells as described in already established protocols of inverted models showed an excessive growth of cellular extensions, as well as complete cells, through the pores of the filter membrane. This effect causes a loss of the monolayer structure, together with a possible reduction of apical/basolateral polarity. Here, we were able to demonstrate an improved cultivation method which resulted in minimal growth through the filter pores.

Formation of a high TEER is a feature characteristic for a functional BCSFB in the in vitro model. It was previously described that cultivation in SFM causes an increase of TEER values in porcine and human models of the BCSFB 
consisting of CP epithelial cells $[15,28]$, which is why the PCP-R cells were also transferred to SFM in this cultivation method. Still, a short exposure to high serum-containing cell growth medium from the basolateral side was essential in order for the development of a suitable TEER overnight when applying the improved "dry-top" cultivation method. In contrast, when prolonged cultivation of PCP-R cells in high serum-containing cell growth medium was carried out, especially from the basolateral side, excessive growth through the filter membrane occurred.

Diverse approaches were originally tested to reduce the undesired growth of PCP-R cells through $3.0 \mu \mathrm{m}$ pores when applying the "conventional" protocol, including collagen-coating of the filter membrane. PCP-R cells grown on collagen-coated membranes did not develop a high TEER in addition to growing extensively through the filter pores. Another technique, which had been described previously, was the application of Parafilm ${ }^{\mathrm{TM}}$ to the filter membrane in order to achieve micropatterns and control the spatial organization of cells [16-18]. Although cellular growth through the pores of the filter membrane was reduced, no high TEER developed, rendering this approach unsuited for our purposes.

We succeeded in preventing cellular growth through filter pores while still obtaining a high TEER and a low flux of Texas Red ${ }^{\mathrm{TM}}$-labeled dextran, by excluding the exposure of serum-containing medium from the basolateral side entirely ("dry-top" cultivation). We suggest that this effect is caused by a rather physical then biochemical mechanism, as has already been concluded by Vandenhaute and colleagues for endothelial cells derived from cord blood hematopoietic stem cells [14].

Finally, we were able to demonstrate a practical application of this improved cultivation method, which resulted in a higher transmigration rate of porcine granulocytes. In this assay, transmigration of granulocytes was higher when either IL- 8 or TNF $\alpha$ were added to the bottom compartment of the cell culture filter insert model. We observed a very efficient transmigration of granulocytes following the stimulation, pointing to a special suitability of the PCP-R cells when analyzing transmigration of porcine immune cells. This is of special interest, since the CP is known to be an active player during immune surveillance of the CNS, and the transmigration of granulocytes is a hallmark of inflammatory events in the brain $[3,10,29,30]$. In this regard, CP epithelial models have been used to study immune cell transmigration during CNS inflammatory events such as bacterial and viral meningitis [12, 31-33]. In addition, functional in vitro models of the BCSFB are of high interest for studying immune cell transmigration during other inflammatory events of the CNS, such as multiple sclerosis $[34,35]$, but also for the analysis of tumor cell entry into the brain [36, 37]. Finally, these models can be employed to study the transport of substances across the $\mathrm{CP}$ epithelium, a feature important for the analysis and development of brain permeable drugs for disease treatment $[7,38]$.

So far, a functional porcine in vitro BCSFB model suitable for analysis of pathogen or immune cell transmigration has only been described for primary PCPEC $[11,12]$. Here we provide a model based on PCP-R cells, which offers the advantages of immortalized cell lines. This model is of interest to study, for example, the mechanisms of meningitis caused by several pathogens. Of high interest would be the Gram-positive bacterium $S$. suis, a zoonotic pathogen that not only causes disease in pigs, but also in humans, with a high incidence found in south-east Asian countries [39, 40]. Other important porcine meningitis-causing pathogens include Haemophilus parasuis and Salmonella species [41-43], although an involvement of the CP has not yet been established. The use of the PCP-R cell-based system could complement studies performed in human BCSFB models and help to discover differences and similarities of disease pathogenesis in pigs and humans.

Acknowledgements The contribution of Marita Meurer and Nicole de Buhr was supported by the DFG grant KO 3552/7-1 provided to Maren von Köckritz-Blickwede, University of Veterinary Medicine, Hannover. We thank Maren von Köckritz-Blickwede for the support in flow cytometry.

\section{Compliance with ethical standards}

Conflict of interest The authors declare that they have no conflict of interest.

Publisher's note: Springer Nature remains neutral with regard to jurisdictional claims in published maps and institutional affiliations.

\section{References}

1. Wolburg H, Paulus W. Choroid plexus: biology and pathology. Acta Neuropathol. 2010;119:75-88.

2. Strazielle N, Ghersi-Egea JF. Choroid plexus in the central nervous system: biology and physiopathology. J Neuropathol Exp Neurol. 2000;59:561-74.

3. Meeker RB, Williams K, Killebrew DA, et al. Cell trafficking through the choroid plexus. Cell Adh Migr. 2012;6:390-6.

4. Wilson EH, Weninger W, Hunter CA. Trafficking of immune cells in the central nervous system. J Clin Invest. 2010;120:1368-79.

5. Engelhardt B, Wolburg-Buchholz K, Wolburg H. Involvement of the choroid plexus in central nervous system inflammation. Microsc Res Tech. 2001;52:112-29.

6. Strazielle N, Ghersi-Egea J-F. In vitro models of the bloodcerebrospinal fluid barrier and their use in neurotoxicological research. Neuromethods. 2011;56:161-84.

7. Ghersi-Egea JF, Strazielle N. Brain drug delivery, drug metabolism, and multidrug resistance at the choroid plexus. Microsc Res Tech. 2001;52:83-88.

8. Lauer AN, Tenenbaum T, Schroten $\mathrm{H}$, et al. The diverse cellular responses of the choroid plexus during infection of the central nervous system. Am J Physiol Cell Physiol. 2018;314:C152-65. 
9. Dinner S, Borkowski J, Stump-Guthier C, et al. A choroid plexus epithelial cell-based model of the human blood-cerebrospinal fluid barrier to study bacterial infection from the basolateral side. J Vis Exp. 2016;111:e54061.

10. Tenenbaum T, Steinmann U, Friedrich C, et al. Culture models to study leukocyte trafficking across the choroid plexus. Fluids Barriers CNS. 2013;10:1.

11. Tenenbaum T, Papandreou T, Gellrich D, et al. Polar bacterial invasion and translocation of Streptococcus suis across the blood-cerebrospinal fluid barrier in vitro. Cell Microbiol. 2009; 11:323-36.

12. Wewer C, Seibt A, Wolburg H, et al. Transcellular migration of neutrophil granulocytes through the blood-cerebrospinal fluid barrier after infection with Streptococcus suis. J Neuroinflammation. 2011;8:51.

13. Schroten M, Hanisch FG, Quednau N, et al. A novel porcine in vitro model of the blood-cerebrospinal fluid barrier with strong barrier function. PLoS ONE. 2012;7:e39835.

14. Vandenhaute E, Drolez A, Sevin E, et al. Adapting coculture in vitro models of the blood-brain barrier for use in cancer research: maintaining an appropriate endothelial monolayer for the assessment of transendothelial migration. Lab Invest. 2016;96:588-98.

15. Schwerk C, Papandreou T, Schuhmann D, et al. Polar invasion and Translocation of Neisseria meningitidis and Streptococcus suis in a novel human model of the blood-cerebrospinal fluid barrier. PLOS ONE. 2012;7:e30069.

16. Javaherian S, O'Donnell KA, McGuigan AP. A fast and accessible methodology for micro-patterning cells on standard culture substrates using Parafilm inserts. PLoS ONE. 2011;6:e20909.

17. Javaherian S, Paz AC, McGuigan AP. Micropatterning cells on permeable membrane filters. Methods Cell Biol. 2014;121:171-89.

18. Paz AC, Javaherian S, McGuigan AP. Tools for micropatterning epithelial cells into microcolonies on transwell filter substrates. Lab Chip. 2011;11:3440-8.

19. Rohnelt RK, Hoch G, Reiss Y, et al. Immunosurveillance modelled in vitro: naive and memory $\mathrm{T}$ cells spontaneously migrate across unstimulated microvascular endothelium. Int Immunol. 1997;9:435-50.

20. Schwerk C, Tenenbaum T, Kim KS, et al. The choroid plexus-a multi-role player during infectious diseases of the CNS. Front Cell Neurosci. 2015;9:80.

21. Seele J, Tauber SC, Bunkowski S, et al. The inflammatory response and neuronal injury in Streptococcus suis meningitis. BMC Infect Dis. 2018;18:297.

22. Federal Ministry of Food and Agriculture B, Germany. Animals used under $\S 7$ (2) of the Animal Protection Act by species. 2017: Page $12 \mathrm{ff}$, Table 11: Use of species in translational and applied research purposes. https://www.bmel.de/SharedDocs/Downloads/ Tier/Tierschutz/Versuchstierdaten2017.pdf?_blob=publicationFile.

23. Meurens F, Summerfield A, Nauwynck H, et al. The pig: a model for human infectious diseases. Trends Microbiol. 2012;20:50-57.

24. Swindle MM, Makin A, Herron AJ, et al. Swine as models in biomedical research and toxicology testing. Vet Pathol. 2012;49:344-56.

25. Gutierrez K, Dicks N, Glanzner WG, et al. Efficacy of the porcine species in biomedical research. Front Genet. 2015;6:293.
26. Aigner B, Renner S, Kessler B, et al. Transgenic pigs as models for translational biomedical research. J Mol Med. 2010;88:653-64.

27. de Buhr N, Neumann A, Jerjomiceva N, et al. Streptococcus suis DNase SsnA contributes to degradation of neutrophil extracellular traps (NETs) and evasion of NET-mediated antimicrobial activity. Microbiology. 2014;160:385-95.

28. Haselbach M, Wegener J, Decker S, et al. Porcine Choroid plexus epithelial cells in culture: regulation of barrier properties and transport processes. Microsc Res Tech. 2001;52:137-52.

29. Ghersi-Egea JF, Strazielle N, Catala M, et al. Molecular anatomy and functions of the choroidal blood-cerebrospinal fluid barrier in health and disease. Acta Neuropathol. 2018;135:337-61.

30. Schwartz M, Deczkowska A. Neurological disease as a failure of brain-immune crosstalk: the multiple faces of neuroinflammation. Trends Immunol. 2016;37:668-79.

31. Dahm T, Frank F, Adams O, et al. Sequential transmigration of polymorphonuclear cells and naive CD3 $+\mathrm{T}$ lymphocytes across the blood-cerebrospinal-fluid barrier in vitro following infection with Echovirus 30. Virus Res. 2017;232:54-62.

32. Steinmann U, Borkowski J, Wolburg H, et al. Transmigration of polymorphnuclear neutrophils and monocytes through the human blood-cerebrospinal fluid barrier after bacterial infection in vitro. $\mathrm{J}$ Neuroinflammation. 2013;10:31.

33. de Buhr N, Reuner F, Neumann A, et al. Neutrophil extracellular trap formation in the Streptococcus suis-infected cerebrospinal fluid compartment. Cell Microbiol. 2017;19:e12649.

34. Vercellino M, Votta B, Condello C, et al. Involvement of the choroid plexus in multiple sclerosis autoimmune inflammation: a neuropathological study. J Neuroimmunol. 2008;199:133-41.

35. Marques F, Sousa JC, Brito MA, et al. The choroid plexus in health and in disease: dialogues into and out of the brain. Neurobiol Dis. 2017;107:32-40.

36. Vandenhaute E, Stump-Guthier C, Lasierra Losada M, et al. The choroid plexus may be an underestimated site of tumor invasion to the brain: an in vitro study using neuroblastoma cell lines. Cancer Cell Int. 2015;15:102.

37. Marz M, Meyer S, Erb U, et al. Pediatric acute lymphoblastic leukemia-Conquering the CNS across the choroid plexus. Leuk Res. 2018;71:47-54.

38. Bernd $\mathrm{A}$, Ott $\mathrm{M}$, Ishikawa $\mathrm{H}$, et al. Characterization of efflux transport proteins of the human choroid plexus papilloma cell line HIBCPP, a functional in vitro model of the blood-cerebrospinal fluid barrier. Pharm Res. 2015;32:2973-82.

39. Huong VT, Ha N, Huy NT, et al. Epidemiology, clinical manifestations, and outcomes of Streptococcus suis infection in humans. Emerg Infect Dis. 2014;20:1105-14.

40. Yu H, Jing H, Chen Z, et al. Human Streptococcus suis outbreak, Sichuan, China. Emerg Infect Dis. 2006;12:914-20.

41. Bouchet B, Vanier G, Jacques M, et al. Interactions of Haemophilus parasuis and its LOS with porcine brain microvascular endothelial cells. Vet Res. 2008;39:42.

42. van der Wolf PJ, Vercammen TJ, Geene JJ, et al. Salmonella typhimurium DT104 septicaemia with meningitis in neonatal piglets. Vet Q. 2001;23:199-201.

43. Wilcock BP, Olander HJ. Neurologic disease in naturally occurring Salmonella choleraesuis infection in pigs. Vet Pathol. 1977;14:113-20. 